\title{
Influence of Acoustical Adaptation on Classroom's Acoustical Enviroment
}

\author{
D. WRÓBLEWSKA ${ }^{a, *}$ AND K. LeO ${ }^{b}$ \\ ${ }^{a}$ Faculty of Civil and Environmental Engineering, Gdańsk University of Technology, Gdańsk, Poland \\ ${ }^{b}$ Faculty of Physics and Applied Mathematics, Gdańsk University of Technology, Gdańsk, Poland \\ A number of objective ana subjective acoustical parameters, referring to the reverberance, and spatial \\ distribution of sound and speech intelligibility, were measured before and after the acoustical adaptation of a \\ classroom. Three configurations of arrangements differing in used materials' sound absorbing coefficient were \\ analyzed. The results indicate the expected improvement of the acoustical environment according to design \\ assumptions.
}

PACS: $43.55 . \mathrm{Dt}$

\section{Introduction}

The contemporary learning process in classrooms is based on verbal activities and involves listening to speech. In classrooms with acoustical discomfort students do not understand the words and the learning process is not effective. Also the presence of noise in the classroom disturbs students activities. Several researches proved the negative impact of noise and the lack of speech intelligibility on not only learning efficiency but also on well-being of students and teachers. Children are impaired by background sounds and teachers suffer from raising their voice level $[1,2]$. On the other hand, positive effects of the classroom acoustical climate improvement on learning and students' behavior are registered in numerous studies [3, 4].

The analyzed classroom has an unsatisfactory acoustical environment for learning because the speech intelligibility is at a poor level (reverberation time (RT) equals about $2.6 \mathrm{~s}$ and speech transmission index (STI) is at a level of $40 \%$ ).

The main aim of this experimental research is to improve acoustical climate of the real classroom by acoustical adaptation. The main problem is the height of the lintel forcing the usage of not typical height of a suspended ceiling ( $15 \mathrm{~cm}$ of the total ceiling height), while the sound absorption is usually given of a typical ceiling, which is 4,10 or $20 \mathrm{~cm}$ of the total ceiling height. This makes it difficult to predict room acoustics by computer simulation. There are two main goals:

- to achieve values of classroom acoustical param-

\footnotetext{
* corresponding author; e-mail:

dominika.wroblewska@wilis.pg.gda.pl
}

eters enabling effective speech communication, learning efficiency and well being according to a chosen set of their preferable values. These parameters are: reverberance, signal to noise ratio and spatial distribution of teacher's speech among students. The acoustical climate is also verified by speech intelligibility parameters such as STI and subjective speech intelligibility test;

- to verify experimentally different combinations of sound-absorption treatment locations as well as their acoustical properties. The absorptive materials are placed mostly on the ceilings, and in this case additionally at the rear wall, which are usually the only adaptable surfaces in classrooms. Locations were chosen to verify the results achieved by for example Sala [5], who indicated that not only the amount of absorption but the localization of material is very important. What is more, the best results in speech intelligibility are achieved while placing absorbing material on both the ceiling and the rear wall.

\section{Acoustical requirements for a small classroom}

The recommended values of different parameters for an unoccupied and furnished classroom vary, depending on the applied standards [6]. The chosen set of preferred values for the classroom is presented in Table $\mathrm{I}$.

\subsection{Analyzed classroom}

The analyzed classroom is in the shape of a typical shoebox of height $3.3 \mathrm{~m}$, length $12.3 \mathrm{~m}$ and width $6.6 \mathrm{~m}$. The volume of the classroom is $266.5 \mathrm{~m}^{3}$. It is furnished with wooden desks fixed in two rows and "loose" (not in fixed position) wooden chairs. The finishing layers 
TABLE I are: walls of painted brickwork, windows, a vinyl floor,

Acoustical requirements for a small classroom.

\begin{tabular}{c|c}
\hline $\begin{array}{c}\text { Parameter } \\
\text { and its measure }\end{array}$ & Required values \\
\hline reverberance, T30 [s] & $\begin{array}{c}0.4-0.8 \\
\text { 20\% less for disabled, } \\
\text { hearing-impaired } \\
\text { and foreign } \\
\text { languages learners }\end{array}$ \\
\hline reverberance, EDT [s] & almost the same as T30 \\
\hline speech intelligibility, & $\geq 60$ [7] \\
STI [\%] & $\begin{array}{c}\geq 0 ; \geq 85 \text { for disabled, } \\
\text { hearing-impaired [8] }\end{array}$ \\
\cline { 2 - 2 } & $\geq 75$ excelent [9] \\
& 60 good; $\geq 45$ fair \\
\hline \multirow{3}{*}{ clarity, C50 [dB] } & $\geq-2[10]$ \\
\cline { 2 - 2 } & $2<$ fair $<+2 ;$ \\
& good $>+2[11]$ \\
\cline { 2 - 2 } & using the correlation \\
& C50=(STI-0.572)/0.028 [12] \\
& $>6.4$ excellent; \\
& $1<$ good $>6.4 ;$ \\
& $-4.4<$ fair $>1$ \\
&
\end{tabular}

blackboards, a wooden door with a small glass window, chairs, desks and a "step desk" made of wood (Fig. 1).

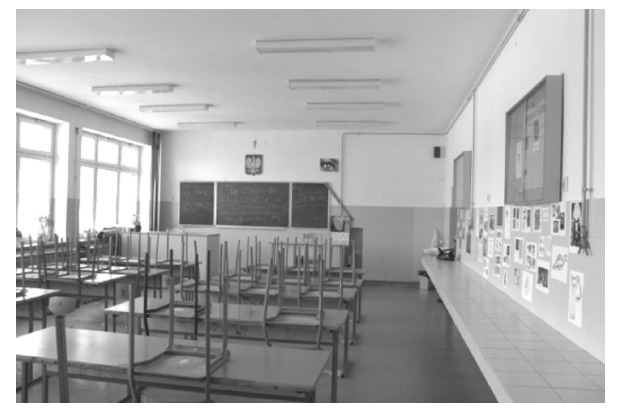

Fig. 1. The interior of the classroom - the view of the front wall with the blackboard.

\subsection{Acoustical adaptation}

Three configurations of acoustical adaptation were chosen for evaluation. Adaptations, presented in Fig. 2, consist of combinations of: suspended ceiling (AD1); suspended ceiling and sound absorbing panels placed on the rear wall (AD2, AD3). The total added sound absorption is presented in Table II.

The surfaces of acoustical materials (Ecophon brand) used in three configurations and their additional sound absorption; TCH - total construction's height.

\begin{tabular}{c|c|c|c|c|c|c}
\hline \hline \multirow{2}{*}{ Material } & \multicolumn{6}{c}{ Configuration } \\
\cline { 2 - 7 } & \multicolumn{2}{|c|}{ AD1 } & \multicolumn{2}{c}{ AD2 } & \multicolumn{2}{c}{ AD3 } \\
\cline { 2 - 7 } & {$\left[\mathrm{m}^{2}\right]$} & $A\left[\mathrm{~m}^{2}\right]$ & {$\left[\mathrm{m}^{2}\right]$} & $A\left[\mathrm{~m}^{2}\right]$ & {$\left[\mathrm{m}^{2}\right]$} & $A\left[\mathrm{~m}^{2}\right]$ \\
\hline master B 200 TCH gamma & 39 & 13 & 39 & 13 & - & - \\
master B 200 TCH alfa & 41 & 35 & 41 & 35 & 41 & 35 \\
advantage A 200 TCH & - & - & - & - & 40 & 32 \\
wall panel $A$ & - & - & 6 & 5 & 6 & 5 \\
sum (\% of total surface) & $80(28 \%)$ & 48 & $86(30 \%)$ & 53 & $87(30 \%)$ & 73 \\
\end{tabular}

\section{Measurement method}

\subsection{Measurements of acoustical parameters}

The measurements were performed in the classroom without students. An omnidirectional source (height of $1.5 \mathrm{~m}$ ) and a microphone (height of $1.2 \mathrm{~m}$ ) were used. Multi length sequence (MLS) technique was utilized in order to obtain impulse responses in six measurement points (Fig. 3). Six measurement points evenly located and one source position in the front of the classroom were used.

\subsection{Measurements of subjective speech intelligibility}

Subjective speech intelligibility was measured using two logatom lists consisting of 100 elements. Each of the participants wrote 200 logatoms, the material was recorded in anechoic conditions. Logatoms were presented in triads in order to achieve a masking process due to apparent reverberation. The presented elements in triads had timing of ordinary speech. Listeners at the age between 14 and 16 years were sitting in groups of four around measurement points. An anechoic recording of the male voice was presented from a pair of directional loudspeakers GENELEC 8020A. Loudspeakers' axes were $120^{\circ}$ apart facing towards the rear corners of the room, located at the omnidirectional source's place.

\section{Measured parameters' values}

Figures 4-8 show spatial distribution of different parameters in measurement points, in three analyzed con- 


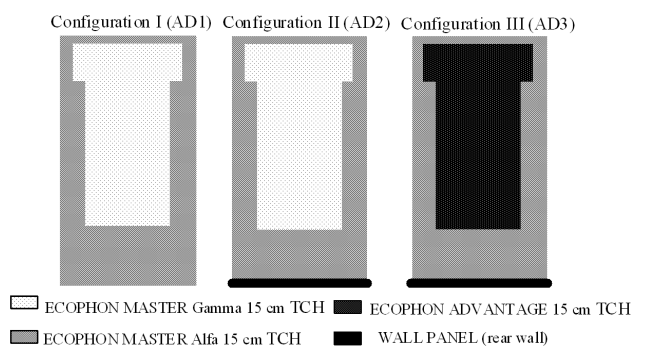

Fig. 2. Configurations of acoustical adaptation. TCH - total construction's height.

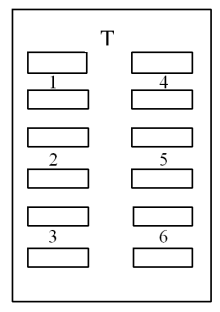

Fig. 3. Location of measurement points (numbers 1-6) at students' desks and T - sound source in front of the classroom.

figurations of acoustic adaptation according to Fig. 2. From far left part - results obtained in the room without acoustic adaptation and equipped with three setups of adaption (AD1, AD2, AD3).

\section{Discussion}

STI values strongly depend on the placement and the amount of absorption material. Before the adaptation, the STI values according to Table I are at the fair level, while after the adaptation at good level for AD1, excellent one for AD2 and AD3. The STI parameter does not depend on the early sound energy, which strongly affects the speech intelligibility. Early energy level (including direct sound) differences in a room result in the higher values of intelligibility for front seats and lower for rear seats in all adapted cases.

Speech tests were carried out for three different acoustical conditions, in which reverberation time varied from $2.5 \mathrm{~s}$ up to $0.5 \mathrm{~s}$ for T30 and the ambient noise level

\begin{tabular}{|c|c|c|c|c|c|c|c|}
\hline a) & $\mathrm{T}$ & b) & T & c) & T & & T \\
\hline 2.56 & 2.62 & 1.11 & 1.09 & 0.54 & 0.56 & 0.52 & 0.52 \\
\hline 2.5 & 2.6 & 1.10 & 1.07 & 0.55 & 0.56 & 0.52 & 0.52 \\
\hline 2.57 & 2.53 & 1.10 & 1.08 & 0.55 & 0.56 & 0.51 & 0.52 \\
\hline & $\square$ & $\square$ & $\square$ & $\square$ & $\square$ & $\square$ & - \\
\hline
\end{tabular}

Fig. 4. Spatial distribution of T30, s: (a) classroom without adaptation; (b) AD1; (c) AD2; (d) AD3.

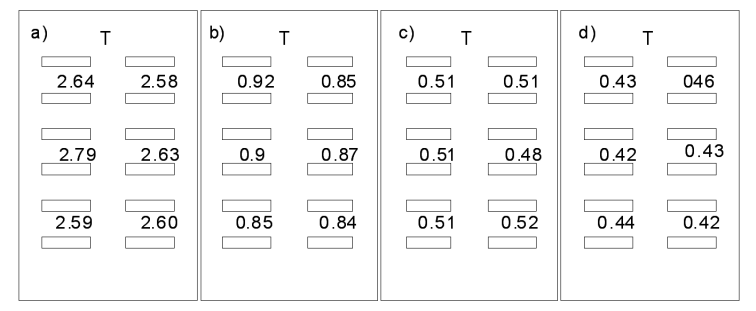

Fig. 5. Spatial distribution of EDT, s: (a) classroom without adaptation; (b) AD1; (c) AD2; (d) AD3.

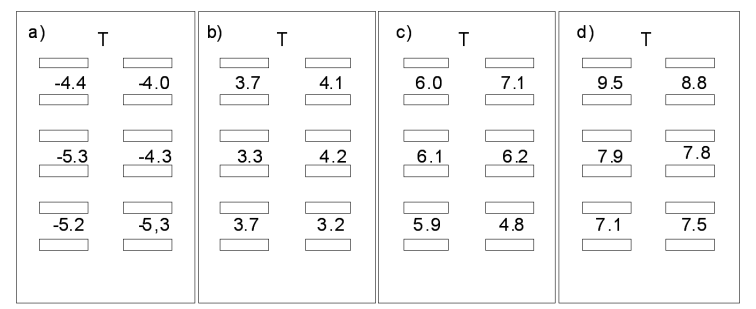

Fig. 6. Spatial distribution of C50, dB: (a) classroom without adaptation; (b) AD1; (c) AD2; (d) AD3.

was constant. Speech intelligibility test results indicate the improvement of that parameter. The average values increase with the amount of sound absorption. Dependence on the position of a seat is strong before adaptation, where direct sound and early energy covers nearly half of the room, while the rest is influenced by late reverberant energy. Reducing reverberation enhances the role of early energy in the whole room. Thus, in the adapted room, intelligibility in back seats is likely to be the same as in the near ones.

The effect of early energy influence on spatial differences in speech intelligibility can be quantified by the early-to-late energy ratio C50, highly correlated with early decay time (EDT) and RT. The strongest spatial differences in speech intelligibility are observed in the room before the adaptation. The values of C50 are at a fair level or less depending on the seat. The significant increase can be observed for AD1 but the excellent results are achieved for AD2 and AD3. The boost to the early reflections given by a reflecting part in the middle of the room (AD2) results in a few percent rise in speech

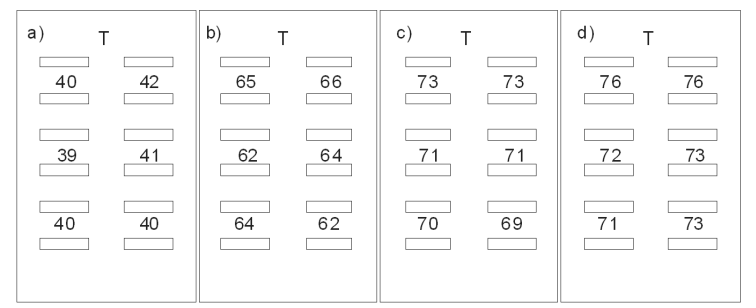

Fig. 7. Spatial distribution of STI, \%: (a) classroom without adaptation; (b) AD1; (c) AD2; (d) AD3. 


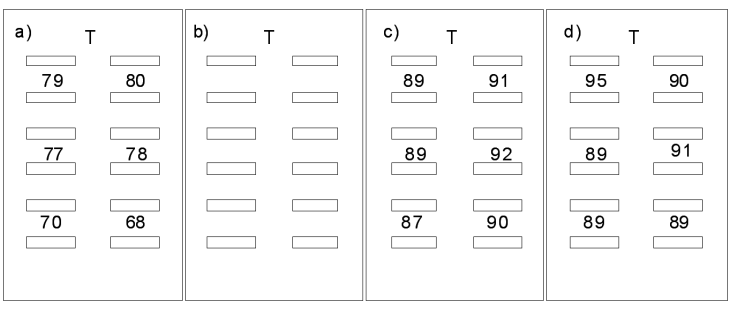

Fig. 8. Spatial distribution of speech intelligibility test \%: (a) classroom without adaptation; (b) AD1 not measured; (c) AD2; (d) AD3.

intelligibility in rear seats (Fig. 8). For AD3 the speech intelligibility scores strongly differ between near and rear seats due to the strong energy difference in the direct and early energy sound.

\section{Conclusions}

The acoustical adaptation in the classroom changed values of acoustical parameters into the desired range. Not only the amount of absorption plays a role, but also its placement is essential. Location on long paths of a sound wave, such as the rear wall, resulted in significant rise in values of speech intelligibility parameters (Figs. 4-8). The rear wall adaptation gave a positive change to both the early and late energy parameters. Thus, the best solution for gaining excellent speech intelligibility is to place the material at least on two surfaces. Covering about $30 \%$ of the total room surface is advisable.

The benefit of early reflections (speech energy arriving within the first $50 \mathrm{~ms}$ after the direct sound) in obtaining adequate speech intelligibility has been confirmed. The early reflections can compensate speech understanding also in cases, where the energy of direct sound is to be low at a more distant listening position. Thus, the layout of absorbing materials should support early reflection energy and a too excessive reverberant sound field should be avoided.

\section{References}

[1] M. Klatte, J. Hellbrück, J. Seidel, P. Leistner, Envir. Behav. 42, 659 (2010).

[2] J.E. Dockrell, B.M. Shield, Brit. Educat. Res. J. 32, 509 (2006).

[3] K. Wall, J. Dockrell, N. Peacey, in: Internoise 2010, Lisbon (Portugal), paper 453, 2010.

[4] G. Iannace, G. Ciaburro, L. Maffei, in: Internoise 2010, Lisbon (Portugal), paper 524, 2010.

[5] E. Sala, , V. Viljanen, Appl. Acoust. 45, 81 (1995).

[6] D. Wróblewska, Acta Phys. Pol. A 118, 186 (2010).

[7] Acoustic Design of Schools. Building Bulletin, United Kingdom 1993.

[8] "SFS 5907: Acoustic classification of space in buildings - Finland", Finland 2004.

[9] A. Abdou, R.W. Guy, J. Acoust. Soc. Am 100, 3215 (1996).

[10] W. Ahnert, W. Schmidt, Akustik in Kulturbauten, Institut für Kulturbauten, Berlin 1980.

[11] W. Cavanaugh, G. Tocci, J. Wilkes, Architectural Acoustics - Principles and Practice, Wiley, New York 2009.

[12] J.S. Bradley, R. Reich, S.G. Norcross, Appl. Acoust. 58, 99 (1999). 